\title{
The New Matrix 4-Chloro- $\alpha$-Cyanocinnamic Acid Allows the Detection of Phosphatidylethanolamine Chloramines by MALDI-TOF Mass Spectrometry
}

\author{
Thorsten Jaskolla, ${ }^{a}$ Beate Fuchs, ${ }^{\mathrm{b}}$ Michael Karas, ${ }^{\mathrm{a}}$ and Jürgen Schiller ${ }^{\mathrm{b}}$ \\ ${ }^{a}$ Cluster of Excellence Macromolecular Complexes, Institute of Pharmaceutical Chemistry, Goethe University \\ Frankfurt, Frankfurt, Germany \\ ${ }^{b}$ Institute of Medical Physics and Biophysics, Faculty of Medicine, University of Leipzig, Leipzig, Germany
}

Phosphatidylethanolamines (PEs) are abundant lipid constituents of the cellular membrane. The amino group of PEs exhibits high reactivity with hypochlorous acid that is generated under inflammatory conditions in vivo. The analysis of the resulting PE mono- and dichloramines is of significant interest since these species represent important mediators of lipid peroxidation. We have shown in a previous communication that mass spectrometric detection of PE chloramines is only possible with ESI MS, whereas MALDI-TOF MS fails to detect these products if standard matrices are used.

In this work we demonstrate that the detection of PE chloramines is also possible by MALDI-TOF MS if 4-chloro- $\alpha$-cyanocinnamic acid is used as matrix. The underlying processes leading to ionization of these species will be discussed in detail. Both, experimental and theoretical studies taking into account possible intramolecular rearrangements were performed to clarify these aspects. (J Am Soc Mass Spectrom 2009, 20, 867-874) (c) 2009 Published by Elsevier Inc. on behalf of American Society for Mass Spectrometry

$\mathrm{H}$ ypochlorous acid $(\mathrm{HOCl})$ is an important reactive oxygen species that is generated under inflammatory conditions from hydrogen peroxide and chloride ions catalyzed by the enzyme myeloperoxidase (MPO) [1]. $\mathrm{HOCl}$ reacts with virtually all biomolecules [2] but the second-order rate constants differ significantly [3]; sulfhydryl and thioether groups exhibit highest reactivity, followed by amino groups. Although olefinic residues of, e.g., lipids also react with $\mathrm{HOCl}$, the corresponding second-order rate constants are relatively small [4].

Since MPO is secreted from the neutrophils [5], the largest amount of $\mathrm{HOCl}$ is generated extracellularly. As a result of the permeability of the cellular membrane for $\mathrm{HOCl}$ [1], the phospholipids of the inner leaflet are also affected by this compound. The low reactive double bonds of the acyl side chains [4] are the only interaction possibility of phosphatidylcholines (PCs) with $\mathrm{HOCl}$, whereas highly reactive amino groups of phosphatidylethanolamines (PEs) allow for much faster reaction [3]. Therefore, PEs are most likely the first molecules that react with $\mathrm{HOCl}$. Mono- and dichloramines are generated as the primary products. Due to the instability of these compounds, further secondary products such as aldehydes and nitriles are also observed

Address reprint requests to Graduate engineer T. Jaskolla, Institute of Pharmaceutical Chemistry, Max-von-Laue-Str. 9, Goethe University Frankfurt, D-60438 Frankfurt, Germany. E-mail: Jaskolla@iachem.de

Conflict of interest statement: The invention of ClCCA as a MALDI matrix was filed as a German patent.
[4]. It has been recently shown that PE-derived chloramines mediate further events of lipid peroxidation [6] and are, therefore, of high interest.

Electrospray ionization mass spectrometry (ESI MS) is an established and widely used tool for the analysis of chlorinated amines and amino acids [7]. PE-derived chloramines were successfully detected as sodiated cations and as deprotonated species with ESI MS [6, 7]. In contrast, matrix-assisted laser desorption and ionization time-of-flight (MALDI-TOF) MS [8] is less suitable for the analysis of chloramines: We have shown in a recent communication that MALDI-TOF MS fails to detect PE-derived chloramines if standard matrices as 2,5-dihydroxybenzoic acid (DHB) or $\alpha$-cyano-4-hydroxycinnamic acid (CHCA) are used [9]. Reactions between the matrix and the chloramines as well as the cleavage of the nitrogen-chlorine bond by UV laser irradiation were both assumed to contribute to this detection problem [9]. Recent investigations have shown that the newly developed matrix 4-chloro- $\alpha$-cyanocinnamic acid (CICCA) easily enables the detection of chlorinated PE species and, therefore, MALDI MS as well as ESI MS are both suitable for the detection of chloramines.

For explaining the beneficial effects of this compound, differences between the used matrices regarding to their reactivity are investigated. We already reported that in comparison with established matrices, ClCCA possesses the lowest matrix proton affinity (PA) known so far, which increases the protonation efficiency of analytes, as, e.g., peptides, phosphopeptides,
(C) 2009 Published by Elsevier Inc. on behalf of American Society for Mass Spectrometry. $1044-0305 / 09 / \$ 32.00$

doi:10.1016/j.jasms.2008.12.028
Published online January 15, 2009 Received October 15, 2008 Revised December 20, 2008 Accepted December 31, 2008 
or proteins [10]. Additionally, we calculated the sodium cation affinity (SCA) of this matrix and further discuss differences of the matrices on the basis of studies regarding their effective plume temperatures and initial ion velocities. Furthermore, we combine these results with closer insights into the underlying mechanism of chlorinated DPPE ionization taking into account protonation and cationization difficulties and possible gasphase rearrangements of these species.

\section{Experimental}

\section{Materials and Reagents}

1,2-Dipalmitoyl-sn-phosphatidylethanolamine (DPPE) was exclusively used in this study because the amino group is the only reactive site within this molecule (regarding to an electrophilic substitution with $\mathrm{HOCl}$ ), and, thus, a simple product spectrum can be expected. 1,2-Dipalmitoyl-sn-phosphatidylcholine (DPPC) lacks a reactive amino group and was therefore additionally used for means of comparison. Both phospholipids are commercially available from Avanti Polar Lipids (Alabaster, MA) and were used without further purification. MilliQ water was prepared by a Millipore (Schwalbach, Germany) water purification system. All other chemicals, salts for buffer preparation, solvents, and the used matrix compounds (CHCA, DHB) were obtained in the highest commercially available purity from SigmaAldrich (Taufkirchen, Germany) and used as supplied.

\section{Synthesis of 4-Chloro- $\alpha$-Cyanocinnamic Acid}

ClCCA was synthesized by a Knoevenagel condensation using cyanoacetic acid (1.05 eq) and 4-chlorobenzaldehyde (1 eq) as educts, piperidinium acetate (0.015 eq) as catalyst and toluene as solvent. Generated water was separated by use of a water trap. The reaction mixture was refluxed for $4 \mathrm{~h}$, cooled and filtered. The crude product was washed with sufficient amounts of distilled water and re-crystallized five times from methanol/water (1:1, vol/vol). Yield after recrystallization: $70.4 \%$ of the theoretical yield. ${ }^{1} \mathrm{H}$ NMR $\left(300 \mathrm{MHz}, \mathrm{DMSO}_{-} \mathrm{d}_{6}\right) \delta=7.66 \mathrm{ppm}(\mathrm{d}, 2 \mathrm{H}, \mathrm{J}=9.4 \mathrm{~Hz})$, $8.02 \mathrm{ppm}(\mathrm{d}, 2 \mathrm{H}, \mathrm{J}=9.8 \mathrm{~Hz}), 8.33 \mathrm{ppm}(\mathrm{s}, 1 \mathrm{H})$ corresponding to an $\mathrm{AA}^{\prime} \mathrm{XX}^{\prime}$ spin system. Accurate mass determination: $\mathrm{M}+\mathrm{H}^{+}$calc. $208.01598 \mathrm{Da}$, detected $208.01584 \pm$ $0.00005 \mathrm{Da}$ (error: $-0.68 \pm 0.22 \mathrm{ppm}$ ).

\section{Computational Methodology}

Visualization and initial building were performed using ChemDraw Pro 10.0 and Chem3D Ultra 10.0 running on a commercial PC system (C2Q Q6600, 3.4 GB RAM). Geometry optimization and computing of the Zero-Point energies (ZPE) of CHCA and CICCA and their sodium cation adducts was performed at the Hartree-Fock level using the triple zeta $6-311++\mathrm{G}(2 \mathrm{~d}, 2 \mathrm{p})$ basis set [11]. The ZPE values calculated at the Hartree-Fock level were corrected by factor 0.91 . Geometry optimization and computing of the ZPE values of $\mathrm{N}, \mathrm{N}$-dichloro-2-aminoethanol and the correlating protonated species was performed by means of density functional theory (DFT) calculations with the hybrid B3LYP method and the triple zeta 6-311++G $(2 \mathrm{~d}, 2 \mathrm{p})$ basis set. The ZPE values calculated with the B3LYP method were corrected by factor 0.9877 [12]. Calculation of all ZPE values was carried out by means of the optimized geometries. For all calculations the Gaussian03 implementation of Chem3D was used.

\section{Reaction Between DPPE and $\mathrm{HOCl}$}

Details of this reaction are given elsewhere [9, 13]. Briefly, vesicles were prepared from DPPE $(1 \mathrm{mM})$ in 50 $\mathrm{mM}$ phosphate buffer $(\mathrm{pH}=6)$ and incubated with a tenfold excess of $\mathrm{HOCl}$ at $37^{\circ} \mathrm{C}$ for $30 \mathrm{~min}$. The concentration of the $\mathrm{HOCl} / \mathrm{NaOCl}$ stock solution was determined at $\mathrm{pH}=12$ using $\varepsilon_{290}=350 \mathrm{M}^{-1} \mathrm{~cm}^{-1}$ [14].

The incubation was stopped by the addition of chloroform/methanol (1:1, vol/vol) in the same volume as the aqueous phase to extract the modified DPPE. The organic phase was separated from the aqueous layer by a Hamilton syringe and immediately used for subsequent MALDI-TOF analysis.

\section{MALDI-TOF Mass Spectrometry}

All MALDI-TOF mass spectra were acquired on a Bruker Autoflex mass spectrometer (Bruker Daltonics, Bremen, Germany) whereas the accurate mass determination of ClCCA was performed on a MALDI LTQ Orbitrap XL (Thermo Fisher Scientific Inc., Waltham, MA) and the determination of the initial velocity of ClCCA was achieved with the help of a Voyager-DE PRO Workstation (Applied Biosystems, Darmstadt, Germany). In the case of DHB a $0.5 \mathrm{M}$ (ca. $77 \mathrm{mg} / \mathrm{mL}$ ) solution in methanol was used [9]. Matrix and sample solution were mixed 1:1 (vol/vol) before deposition onto the MALDI target.

CHCA and ClCCA were also used as matrices. CHCA was used as $10 \mathrm{mg} / \mathrm{mL}$ solution in $70 / 30$ acetonitrile $(\mathrm{ACN}) / 0.3 \%$ trifluoroacetic acid (TFA) (vol/ $\mathrm{vol})$, whereas a $30 \mathrm{mM}(6.21 \mathrm{mg} / \mathrm{mL})$ solution of ClCCA was prepared in $70 / 30 \mathrm{ACN} / 1.5 \%$ TFA (vol/vol).

Laser desorption mass spectra of the pure matrix compounds were recorded in the positive- (a) and negative ion (b) mode for the sake of comparison and added as Supplemental Information (SI) Figure 1, which can be found in the electronic version of this article. A volume of $0.5 \mu \mathrm{L}$ of the individual matrix solution was spotted onto a polished stainless steel target, followed by the addition of $0.5 \mu \mathrm{L}$ of the above mentioned organic DPPE extracts or a pure DPPE solution for reference purposes, respectively, and air-dried. Slight alterations of this sample preparation regime did not result in significant changes of spectral quality.

The used Autoflex MALDI-TOF mass spectrometer utilizes a pulsed nitrogen laser, emitting at $337 \mathrm{~nm}$. The extraction voltage was $20 \mathrm{kV}$ and gated matrix suppression was applied to prevent the saturation of the 
detector by matrix ions. 128 single laser shots were averaged for each mass spectrum. The laser strength was kept about $10 \%$ above the threshold fluence, defined as the minimum laser intensity required to detect any analyte signals, to obtain an optimum signal-tonoise $(\mathrm{S} / \mathrm{N})$ ratio. To enhance the spectral resolution all spectra were acquired in the reflector mode using delayed extraction (DE) conditions. A more detailed methodological description of MALDI-TOF MS of phospholipids is given in [8].

The mass of ClCCA $+\mathrm{H}^{+}$was calculated as the average value of six measurements. The parameters for the accurate mass determination were as follows: analyzer, FTMS; resolution, 100,000; laser fluence, $15 \mu \mathrm{J}$; scans/spectrum, 30.

\section{Measurement of the Initial Velocity of ClCCA}

In a linear two stage acceleration TOF system the initial velocity of the ablated ions can be determined from the slope of the linear regression of total flight time versus DE time [15, 16]. Device parameters were set to identical values as given in [17]. For each DE time the average flight time of the protonated analyte ions was determined using data from six individual measurements accumulated of 50 shots each. Peak labeling was done at $50 \%$ peak centroid after smoothing the spectra using the method of Savitzky-Golay at 251 data points.

\section{Results and Discussion}

As has been known for several years [18], phospholipids can be analyzed with MALDI-TOF MS by using a large variety of different matrix compounds. Positive $(\mathbf{a}-\mathbf{c})$ and negative $(\mathbf{d}-\mathbf{e})$ ion mode MALDI-TOF mass spectra of DPPE $\left(\mathrm{m} / \mathrm{z}_{\text {neutral, monoiso }}=691.5\right)$ recorded with different matrix compounds are shown in Figure 1 for means of comparison. It is evident that DHB (1a) as well as ClCCA (1c) provide spectra of comparable quality although peak intensities differ slightly. This

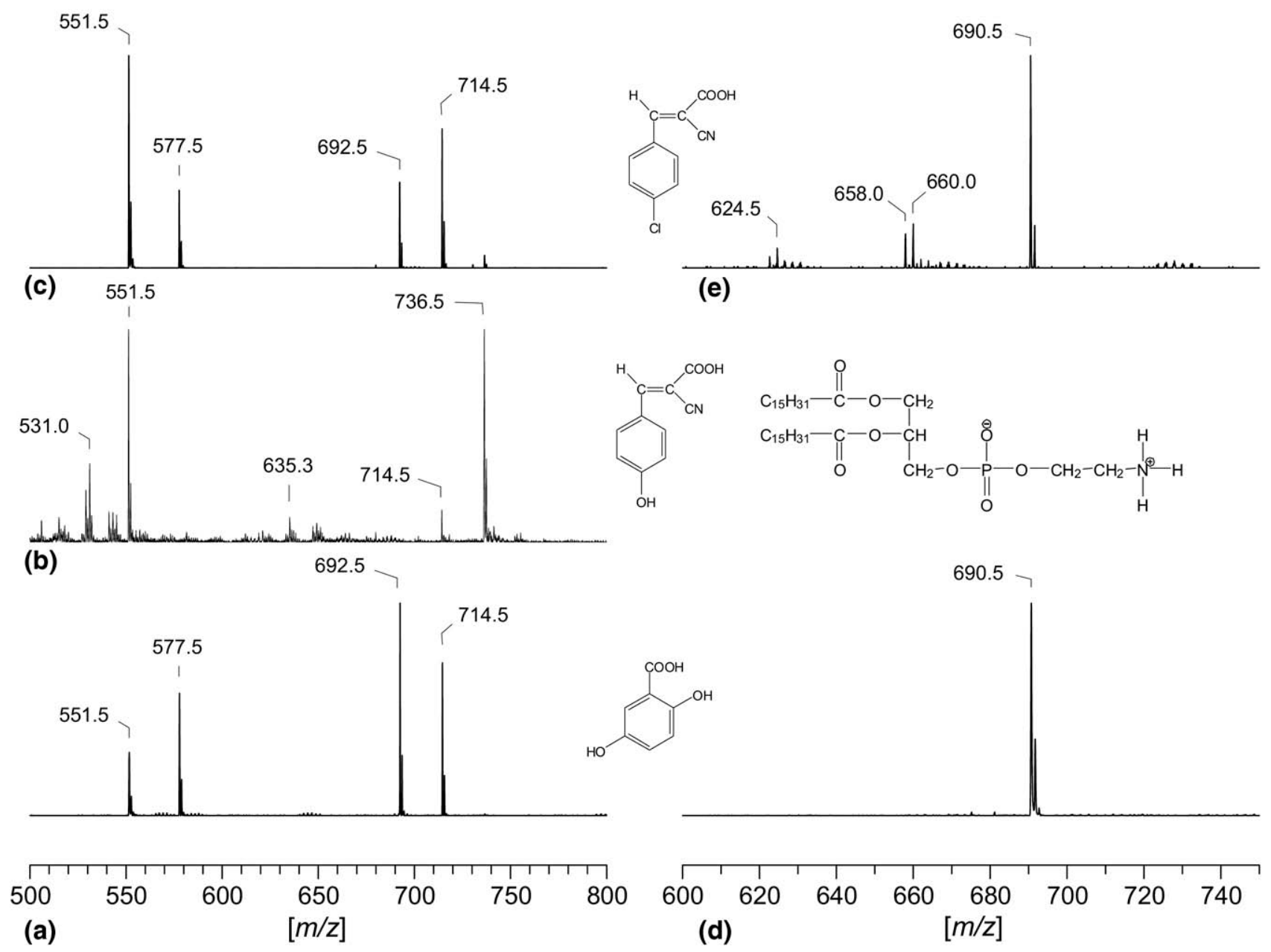

Figure 1. Positive (a)-(c) and negative (d), (e) ion mode MALDI-TOF mass spectra of DPPE recorded in the presence of different matrices: $\operatorname{DHB}(\mathbf{a}),(\mathbf{d}), \mathrm{CHCA}(\mathbf{b})$, and ClCCA (c), (e) were used. The most intense peaks are marked with the $\mathrm{m} / \mathrm{z}$ value and the chemical formulae of the different matrix compounds and the analyte (DPPE) are also shown. It was not possible to record a convincing negative ion mode spectrum of DPPE with CHCA as matrix. Please also note the lack of protonated DPPE and the fragment ion at $m / z=692.5$ and 577.7, respectively, if CHCA is used as matrix. 
particularly concerns the intensity of the fragment ion at $m / z=551.5$ and the intensity ratio between the protonated species $(m / z=692.5)$ and the sodium cation adduct $(m / z=714.5)$ of DPPE [19]. The spectral quality of DPPE in the presence of CHCA (1b) is obviously much lower regarding the achievable $S / N$ ratio. However, it is remarkable that using CHCA as matrix the $\mathrm{DPPE}+\mathrm{H}^{+}$ion at $m / z=692.5$ is completely absent, whereas the double sodium cation adduct [DPPE $-\mathrm{H}+$ $2 \mathrm{Na}]^{+}$at $m / z=736.5$ exhibits a very strong intensity. This ion is generated only in small yields if other matrices are used. It is furthermore remarkable that when using CHCA as matrix the fragment ion at $\mathrm{m} / \mathrm{z}=$ 577.5 is also missing. This indicates that this fragment ion is generated exclusively from the protonated species of DPPE, but not from the sodium cation adduct(s) and this will be discussed below in more detail. A similar fragmentation induced by unstable protonated ions was already reported in the case of triacylglycerols [20]. DPPE is also detectable as deprotonated negative ion $(\mathbf{1 d}, \mathbf{e})$ if $\mathrm{DHB}$ or CICCA are used, but not in the presence of CHCA (spectrum not shown). Obviously, $\mathrm{DHB}$ is the matrix of choice for negative ion detection, because ClCCA gives a higher matrix background. Nevertheless, the focus of this paper will be on the positive ion mode spectra.

One problem related to the analysis of the chloramines of DPPE is their limited stability, because they tend to eliminate $\mathrm{HCl}$ under formation of imines and nitriles $[9,21]$. However, the initially generated products are sufficiently stable, at least for one hour, which is sufficiently long for MALDI-TOF MS analysis. Relatively high amounts of $\mathrm{HOCl}$ were used to generate exclusively the dichloramine of DPPE $\left(\mathrm{Cl}_{2} \mathrm{DPPE}\right)$. The dichloramine is more suitable from the analytical point of view since the monochloramine exhibits a lower stability, because it disproportionates easily into the PE and the dichloramine [21].

Figure 2 shows the positive ion mode MALDI-TOF mass spectra of DPPE after the treatment with a tenfold excess of $\mathrm{HOCl}$ recorded with different matrix compounds. It is evident that the spectra recorded in the presence of DHB (2a) and CHCA (2b) on the one hand and CICCA on the other hand (2c) differ significantly. As already shown in Figure 1, remaining underivatized DPPE can be detected as protonated species both by DHB and ClCCA $(\mathbf{2} \mathbf{a}, \mathbf{c})$ and as sodiated species also with CHCA (2b). The expected mono- and dichloramine derivatives cannot be detected even in traces using the common matrices DHB (2a) or CHCA (2b), but intense signals of the singly and doubly sodiated adducts of dichloramine at $\mathrm{m} / \mathrm{z}=782.5$ and $804.5 \mathrm{(m} / \mathrm{z}$ values refer to the ${ }^{35} \mathrm{Cl}$-isotope signals in all cases) are detectable when ClCCA is used (2c). However, no protonated ions of the dichloramine can be observed. As a result, the lack of the dichloramine peaks in (2a) and $(2 \mathbf{b})$ cannot be explained by a reaction of the chloramine with the matrix or the depletion of the dichloramine upon UV laser irradiation [9].

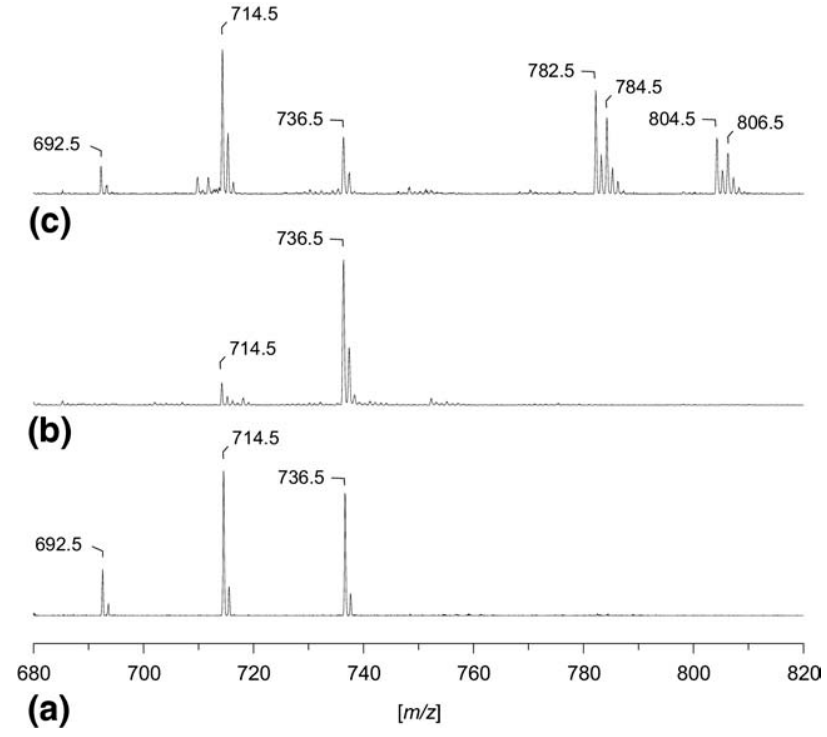

Figure 2. Positive ion mode MALDI-TOF mass spectra of DPPE after treatment with a 10 fold excess of $\mathrm{HOCl}$. Spectra were recorded with different matrices: DHB (a), CHCA (b), and ClCCA (c) were used. Please note the exclusive detectability of the chloramine of DPPE in the case of ClCCA. For details see text.

The detection of the sodiated $\mathrm{Cl}_{2} \mathrm{DPPE}$ adducts cannot be due to possible $\mathrm{Na}^{+}$-contaminations of the used ClCCA matrix, since addition of sufficiently high amounts of $\mathrm{NaCl}$ to CHCA and DHB preparations did not enable the detection of these adducts (data not shown). Due to the low proton affinity (PA) of ClCCA, this compound was already successfully used for the analysis of protonable analytes like proteins and peptides and provided spectra of superior quality in comparison to common MALDI matrices [10], but this new matrix was not yet used for the detection of chloramines. It was supposed that because of the clear detection of sodium cation adducts of the DPPE dichloramine not only the PA of ClCCA but also the sodium cation affinity (SCA) is lowered compared with common MALDI matrices. Therefore, the SCA values for ClCCA and CHCA were computed as the energy difference between the matrix $+\mathrm{Na}^{+}$complex (SI Figure 2) and the isolated species (SI Figure 3). For this purpose the energetically most favorable binding site of the sodium cation on the matrix molecules and the lowest energy isomers of the neutral matrix structures were calculated using the Hartree-Fock level with the $6-311++G(2 d, 2 p)$ basis set. The most stable conformation of the $\mathrm{CHCA}+\mathrm{Na}^{+}$complex was found to involve sodium chelation between the carbonyl oxygen of the trans acid, and the nitrogen atom (SI Figure 2) [22]. For ClCCA different binding sites including sodium cation binding at the chloro substituent were tested. The attachment site with the lowest energy content was found to be at the same position as for CHCA. The SCA of CHCA was calculated to be $192.5 \mathrm{~kJ} / \mathrm{mol}$ (SI Table 1), which is in good agreement with the experimental value of about $190.5 \mathrm{~kJ} / \mathrm{mol}$ reported [23]. The SCA for 
ClCCA was calculated to be $177.9 \mathrm{~kJ} / \mathrm{mol}$ and is thus $14.6 \mathrm{~kJ} / \mathrm{mol}$ lower in energy than the corresponding value for CHCA ( $>$ SI Table 1). The lower SCA of ClCCA can be explained by the strong electron withdrawal (-I) effect of the chloro substituent which leads to a lower stabilization of the sodiated complex compared to CHCA with the electron donating hydroxyl substituent in para position of the aromatic ring. Therefore, the CICCA SCA value is still significantly higher than the corresponding value of DHB (about 144.4 $\mathrm{kJ} / \mathrm{mol}$ ) [22]. Since not even traces of the dichloramine of DPPE are detectable using DHB (cf. trace 2a), further differences between CHCA and DHB on the one hand and $\mathrm{ClCCA}$ on the other hand must be responsible for the observed differences. Because ClCCA exhibits the lowest PA of all known MALDI matrices, the question arises whether the low PA promotes the formation of the sodium cation adducts. Unlike $\mathrm{Cl}_{2} \mathrm{DPPE}+\mathrm{Na}^{+}$ adducts, however, the protonated species still cannot be detected using ClCCA as matrix. Therefore it was of interest, whether the basicity of $\mathrm{Cl}_{2} \mathrm{DPPE}$ is too low for protonation by the available matrices because of the strong electron-withdrawing chloro substituents which decrease the PA of the basic amino group. For this purpose the PA of the energetically lowest conformer of the dichlorinated ethanolamine head group of $\mathrm{Cl}_{2} \mathrm{DPPE}$ was calculated using the B3LYP method with the $6-311++\mathrm{G}(2 \mathrm{~d}, 2 \mathrm{p})$ basis set (SI Figure 4$)$. The PA was calculated to be $789.9 \mathrm{~kJ} / \mathrm{mol}$ and is, therefore, about 42 $\mathrm{kJ} / \mathrm{mol}$ lower compared to the experimentally determined PA value of $832 \mathrm{~kJ} / \mathrm{mol}$ for ClCCA [10]. As a result, no protonation of $\mathrm{Cl}_{2} \mathrm{DPPE}$ can occur which explains the missing of the corresponding $\mathrm{Cl}_{2} \mathrm{DPPE}+$ $\mathrm{H}^{+}$signals.

In contrast, however, protonation can occur in the case of deprotonated DPPE; the phosphate hydroxyl group exhibits a high acidity and can be easily deprotonated. This is supported by a strong [DPPE - H] $]^{-}$ion signal $(m / z=690.5$, cf. Figure 1$)$ and by the formation of $[\mathrm{DPPE}-\mathrm{H}+2 \mathrm{Na}]^{+}(m / z=736.5$, cf. Figure 1$)$ and $\left[\mathrm{Cl}_{2} \mathrm{DPPE}-\mathrm{H}+2 \mathrm{Na}\right]^{+}(\mathrm{m} / \mathrm{z}=804.5 \mathrm{cf}$. Figure 2$)$. Furthermore, it can be rationalized that neutral and therefore not detectable [DPPE $-\mathrm{H}+\mathrm{Na}$ ] and its chlorinated derivatives will be generated within the matrix crystals or the matrix plume.

Deprotonated chlorinated DPPE could not be detected, probably because of lacking stability without the $\mathrm{Na}^{+}$counterion. It is assumed that these species undergo further stabilization by abstraction of chloride anions which leads to formation of the corresponding imines and nitriles, respectively [9].

Proton transfer from highly reactive $\mathrm{ClCCA}+\mathrm{H}^{+}$to the neutral $\left[\mathrm{Cl}_{2} \mathrm{DPPE}-\mathrm{H}+\mathrm{Na}\right]$ species will be possible and exothermic since in this case the most basic position is located at the deprotonated phosphate group, resulting in formation of the detectable sodium cation adduct $\mathrm{Cl}_{2} \mathrm{DPPE}+\mathrm{Na}^{+}$. The missing detectability of sodiated $\mathrm{Cl}_{2} \mathrm{DPPE}$ ions using CHCA or DHB as matrix could be due to a decreased basicity of the deprotonated phos- phate group coordinated by a sodium cation. The generation of the correlating doubly sodium cationized $\mathrm{Cl}_{2}$ DPPE ion seems to be more complicated: Simple sodium cation adduct formation to neutral $\left[\mathrm{Cl}_{2} \mathrm{DPPE}-\mathrm{H}+\mathrm{Na}\right]$ cannot be responsible for the generation of this ion, since in this case this species would be also detectable with other matrices than ClCCA exhibiting comparable or lower SCA values. Assuming an analogous process of ion formation as for the singly sodiated ion species would require a proton transfer to the neutral species $\left[\mathrm{Cl}_{2} \mathrm{DPPE}-2 \mathrm{H}+2 \mathrm{Na}\right]$, which appears chemically rather improbable, since $\mathrm{Cl}_{2} \mathrm{DPPE}$ has only one acidic functional group. Nevertheless, using 1,2dipalmitoyl-sn-phosphatidylcholine (DPPC) with a quaternary trimethylammonium group exhibiting a permanent positive charge, the corresponding deprotonated species can be detected using CICCA as matrix: In Figure 3 the positive- (a) and the negative ion (b) mode MALDITOF mass spectrum of DPPC is shown. As expected, the positive ion mode spectrum shows the protonated ion species and the sodium cation adduct at $\mathrm{m} / \mathrm{z}=734.5$ and 756.5, respectively. This is a common feature of all PC species [8]. Surprisingly, the intact DPPC was also detected as deprotonated ion at $m / z=732.5$. This is, to our best knowledge, the very first report about the detection of a PC molecule as negative ion by MALDITOF MS. This finding was actually unexpected as the DPPC lacks (in contrast to DPPE) acidic functional groups because the negative charge of the phosphate group is required to compensate the positive charge of the quaternary trimethylammonium group. In addition, there are also intense negative ion signals $(\mathbf{3 b})$ at $\mathrm{m} / \mathrm{z}=$ 717.5 and 673.5 corresponding to the loss of a methyl group and of trimethylamine, respectively. Consequently, a second deprotonation of DPPC seems to be possible, which cannot simply occur in solution but must be due to gas-phase processes. It seems likely that similar processes are also possible in the case of $\mathrm{Cl}_{2} \mathrm{DPPE}$ which may potentially explain the generation of the doubly sodiated species. For explaining the second deprotonation step, the assumption of an electron capture at the carbonyl-carbon atom of one of the acyl-side chains resulting in the generation of a radical anion seemed reasonable (SI Figure 5a). Subsequent elimination of a hydrogen-radical would lead to the formation of an enolate-anion stabilized by mesomerism, (SI Figure 5b). To verify this possibility, ethylpalmitate as an integral part of the DPPC and DPPE structure was analyzed with $\mathrm{ClCCA}$ as matrix (SI Figure 6). The amount used (50 pmol) was sufficiently high to clearly detect ethylpalmitate as radical cation in the positive ion mode $(\mathrm{m} / \mathrm{z}=284.27)$. In the negative ion mode neither the radical anion nor the deprotonated species could be detected (SI Figure 6), excluding the possibility of a participation of the fatty acyl side chains in analyte deprotonation. From these data we suggest that a rearrangement of DPPC and DPPE can explain the second deprotonation step, which is shown in Figure 4 . The protonated head group $\mathrm{NR}_{3}{ }^{+}(4 \mathbf{a})$ exhibits 


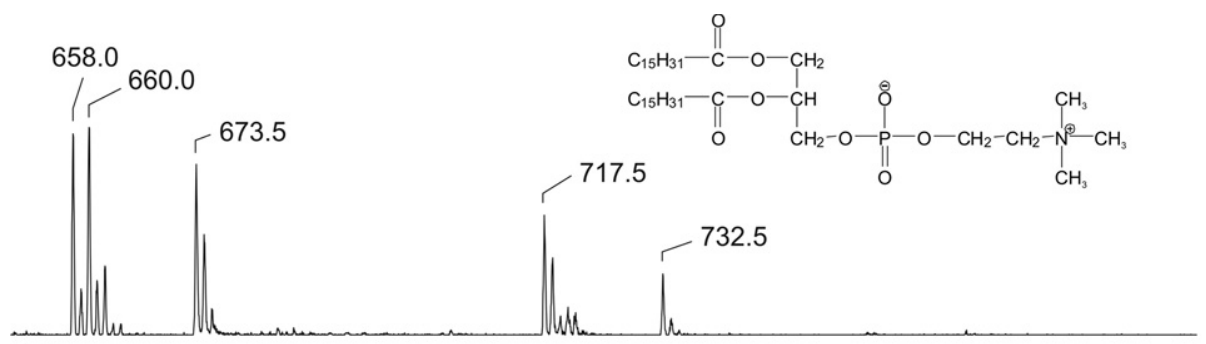

(b)

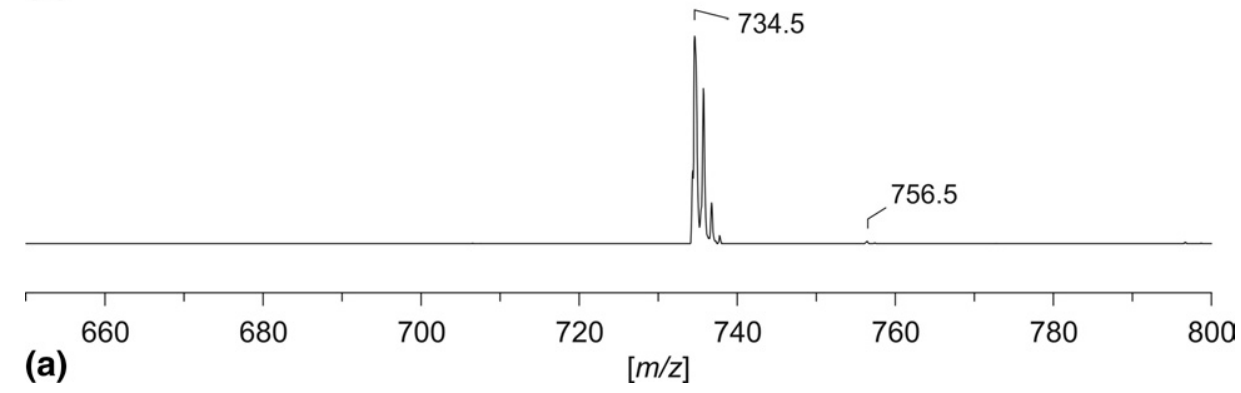

Figure 3. Positive- (a) and negative-ion (b) mode MALDI-TOF mass spectra of DPPC, the structure of which is shown in the upper part of the figure. Spectra were recorded with ClCCA as matrix. Peaks are labeled with their corresponding $\mathrm{m} / \mathrm{z}$ values. Please note that the peaks at $\mathrm{m} / \mathrm{z}=658.0$ and 660.0 are caused by the CICCA matrix and have nothing to do with the DPPC analyte.

an electron-withdrawing effect which weakens the carbon-hydrogen bond of the neighbored methylene group. As a result, a methylene-proton can be transferred to the negatively polarized oxygen. A second bond breaking between the oxygen and the glycerol methylene group will cause a rearrangement under formation of a terminal phosphate group with two acidic functions $(4 \mathbf{b})$. Independently of the respective head group, this species can be easily deprotonated twice which may simply explain the generation of the detected deprotonated DPPC as well as the detected doubly sodium cationized $\mathrm{Cl}_{2} \mathrm{DPPE}$ ion, for which a doubly deprotonated precursor ion was assumed.

Moreover, a second rearrangement can explain further experimental observations; the methylene group connecting the terminal phosphate with the rest of the molecule is acidified by the electron withdrawing effects of both the phosphate group and the positively charged amine $(4 \mathbf{b})$. As a result, a proton shift to the phosphate group with elimination of phosphoric acid and the neutral tertiary amine can occur (4c). Both, protonated amine and deprotonated phosphoric acid can be detected in the corresponding MS spectra (data not shown). The detection of the dihydrogenphosphate cannot be explained without taking into account molecular rearrangements. This rearrangement is only possible, if the amino group is positively charged, which explains, while in the case of DPPE the remaining positively charged diacylglycerol-like compound at $\mathrm{m} / \mathrm{z}=577.5$ can only be observed when the protonated DPPE is also detectable (cf. Figure 1). In the case of $\mathrm{Cl}_{2}$ DPPE protonation of the amine is not possible because of the electron-withdrawing chloro substituents.
Therefore, elimination of dichloramine and phosphoric acid cannot occur, which explains the lack of $\mathrm{m} / \mathrm{z}=$ 577.5 in the case of chlorinated DPPE. Since the rearrangement of $\mathrm{Cl}_{2} \mathrm{DPPE}$ is restricted to $4 \mathrm{~b}$ without further fragmentation, the amount of $4 \mathbf{b}$ will be higher than for DPPE or DPPC. This could explain the increased formation of doubly sodiated $\mathrm{Cl}_{2} \mathrm{DPPE}$ (cf. Figure 2c) compared with underivatized DPPE (cf. Figure 1c). The initial structure of the remaining diacylglycerol-like compound (4c) is not stable and can undergo the further rearrangements A and B illustrated in Figure 4. Both ions can be detected in the mass spectrum, whereas the ion at $\mathrm{m} / \mathrm{z}=551.5$ can also be generated by bond breaking between the phosphate oxygen and the acylated glycerol residue of the intact phospholipid (4a). This explains why this ion is also detectable when the protonated phospholipid cannot be detected (cf. Figure 1b). The assumed rearrangement cannot be limited to CICCA, since the resulting fragment ion at $m / z=577.5$ can be detected also with DHB (cf. Figure 1a, 1c). Accordingly, compound $4 \mathrm{~b}$ must also be generated with this matrix. Nevertheless, doubly deprotonated DPPC as one of the assumed rearrangement products can only be detected with ClCCA. A possible explanation for this discrepancy is that only a minor part of the respective phospholipid undergoes a rearrangement, resulting in a low amount of $\mathbf{4 b}$, which furthermore can undergo further decomposition steps. The degree of analyte fragmentation in the presence of a certain matrix is strongly dependent on the corresponding effective temperature of the analyte [24, 25]. Matrices with high effective temperatures typically cause intense analyte fragmentation. At laser fluences 


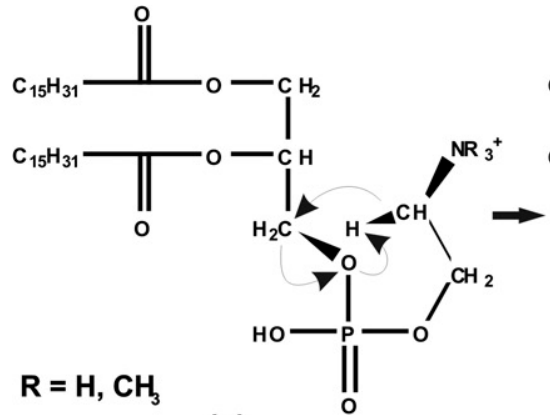

(a)

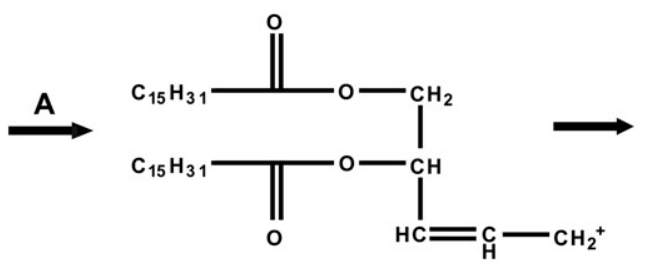

(b)
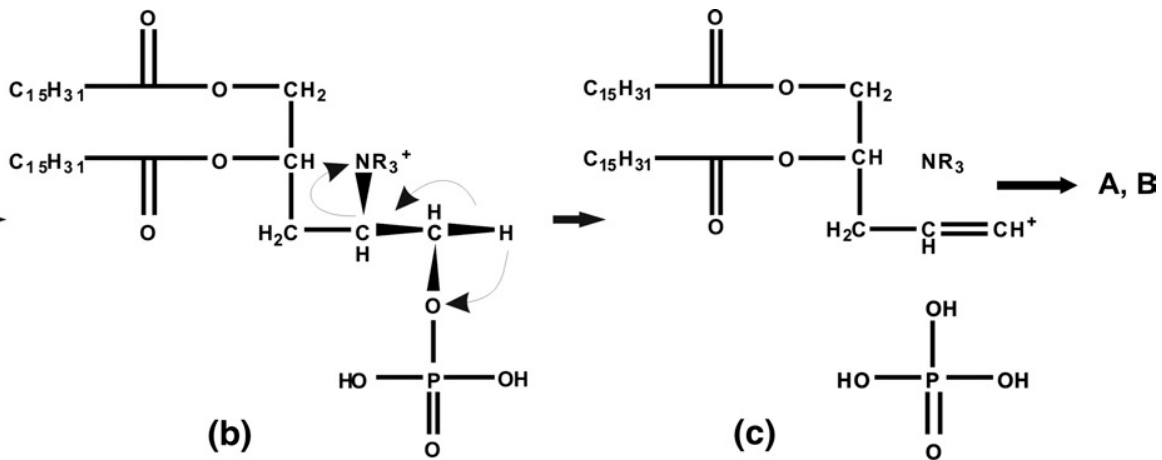

(c)
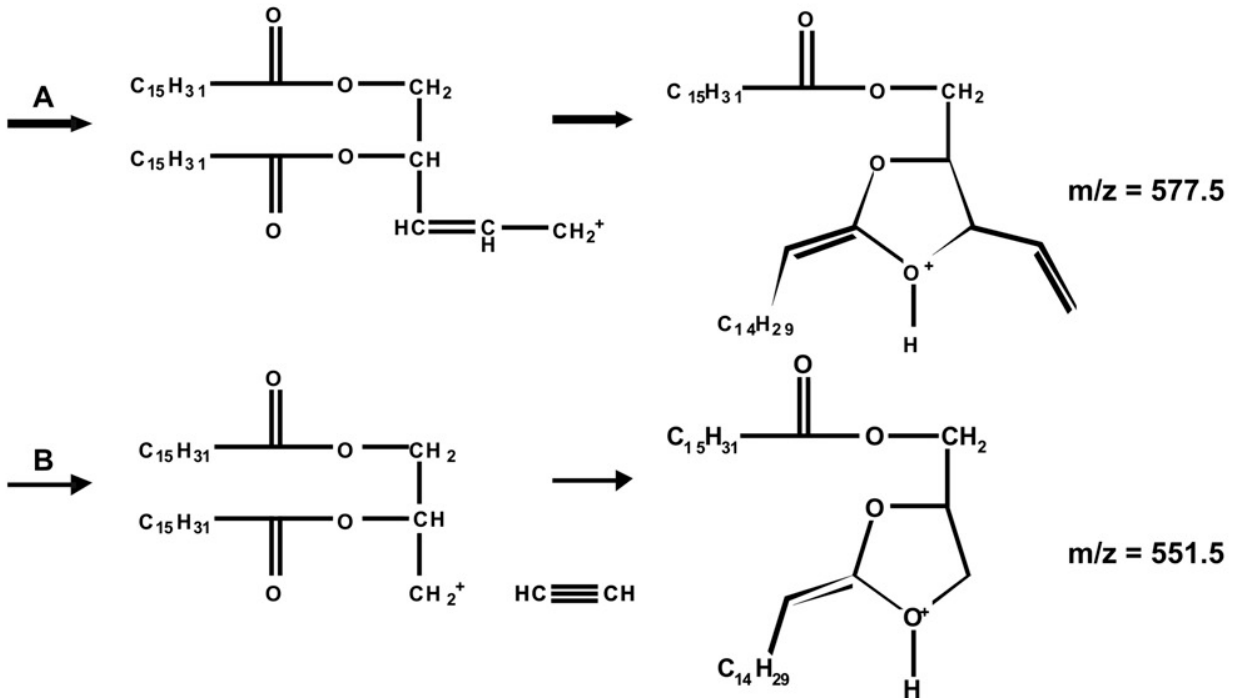

$\mathrm{m} / \mathrm{z}=551.5$<smiles>O=P(O)(O)O</smiles><smiles>[AsH3][AsH2]=C1CCCCC1</smiles>

Figure 4. Suggested rearrangement of DPPE capable of explaining both assumed deprotonation steps. The initially generated ions stabilize themselves by rearrangement A or B under generation of the ions detectable in the positive ion mode mass spectra at $m / z=577.5$ and 551.5 . For details referring to the rearrangement see text.

slightly above the threshold fluence, ClCCA exhibits effective temperatures about 100 to $200 \mathrm{~K}$ higher than in the case of DHB [10, 24, 25]. Another matrix parameter that has been proposed to correlate with analyte internal energy is the initial velocity of the desorbed molecules and ions [15, 26]. It is assumed that a correlation exists between the internal energy of analytes and their translational energy, or the velocity of the expanding plume, respectively. The higher the velocity of the expanding plume is, the lower the internal energy will be, resulting in a stabilization of the desorbing analytes. For DHB an initial velocity of $543 \mathrm{~m} / \mathrm{s}$ was reported [15], whereas the corresponding value of ClCCA is about $350 \mathrm{~m} / \mathrm{s}$. It is straightforward to assume that due to both effects also the intensity of analyte fragmentation is increased in the case of ClCCA, resulting in a stronger generation of $4 \mathbf{b}$, which may allow for the detection of, e.g., deprotonated DPPC in the negative ion mode. This is further supported by means of the intensity ratio of the fragment ion at $\mathrm{m} / \mathrm{z}=577.5$, which is exclusively formed by the rearrangement shown in Figure 4, and its precursor ion at $\mathrm{m} / \mathrm{z}=692.5$. This ratio exhibits a reproducible value of about 0.6 for DHB, while it rises to about 1 in the case of ClCCA (cf. Figure 1). Also, the absolute intensity of the fragment ion at $\mathrm{m} / \mathrm{z}=551.5$ increases strongly when ClCCA is used (cf. Figure 1), indicating a clearly higher fragmentation efficiency.

\section{Conclusions}

The detection of deprotonated DPPC using ClCCA as matrix is a strong indication for an intramolecular gas-phase rearrangement of phospholipids exhibiting ethanolamine head groups. This rearrangement seems to be a necessary requirement for the ionization of chlorinated PE species. Although some details of the ion generation processes still remain unknown and have to be further investigated in more detail, the most important result of the present investigation is that chlorinated PE species are only detectable if a suitable matrix as ClCCA is selected. This makes MALDI-TOF MS coequal to ESI regarding the detection of chloramines.

\section{Acknowledgments}

The authors acknowledge financial support from the German Research Council (DFG Schi 476/5-1 and FU 771/1-1) and the Federal Ministry of Education and Research (grant BMBF 0313836). 


\section{Appendix A. Supplementary Material}

Supplementary material associated with this article may be found in the online version at doi:10.1016/ j.jasms.2008.12.028.

\section{References}

1. Schiller, J.; Fuchs, B.; Arnhold, J.; Arnold, K. Contribution of reactive oxygen species to cartilage degradation in rheumatic diseases: Molecular pathways, diagnosis, and potential therapeutic strategies. Curr. Med. Chem. 2003, 10, 2123-2145.

2. Winterbourn, C. C. Comparative reactivities of various biological compounds with myeloperoxidase-hydrogen peroxide-chloride, and similarity of the oxidant to hypochlorite. Biochim. Biophys. Acta. 1985, 840, 204-210.

3. Pattison, D. I.; Hawkins, C. L.; Davies, M. J. Hypochlorous acidmediated oxidation of lipid components and antioxidants present in low-density lipoproteins: Absolute rate constants, product analysis, and computational modeling. Chem. Res. Toxicol. 2003, 16, 439-449.

4. Fuchs, B.; Schober, C.; Richter, G.; Nimptsch, A.; Sü $\beta$, R.; Schiller, J. The Reactions between $\mathrm{HOCl}$ and differently saturated phospholipids: Physiological relevance, products and methods of evaluation. Mini-Rev. Org. Chem. 2008, 5, 254-261.

5. Palmblad, J. The role of granulocytes in inflammation. Scand. I. Rheumatol. 1984, 13, 163-172

6. Kawai, Y.; Kiyokawa, H.; Kimura, Y.; Kato, Y.; Tsuchiya, K.; Terao, J. Hypochlorous acid-derived modification of phospholipids: Characterization of aminophospholipids as regulatory molecules for lipid peroxidation. Biochemistry. 2006, 45, 14201-14211.

7. Raftery, M. J. Detection and characterization of N- $\alpha$-chloramines by electrospray tandem mass spectrometry. Anal. Biochem. 2007, 366, 218 227.

8. Schiller, J.; Süß, R.; Arnhold, J.; Fuchs, B.; Leßig, J.; Müller, M.; Petković, M.; Spalteholz, H.; Zschörnig, O.; Arnold, K. Matrix-assisted laser desorption and ionization time-of-flight (MALDI-TOF) mass spectrometry in lipid and phospholipid research. Prog. Lipid Res. 2004, 43, $449-488$.

9. Richter, G.; Schober, C.; Süß, R.; Fuchs, B.; Birkemeyer, C.; Schiller, J. Comparison of the positive and negative ion electrospray ionization and matrix-assisted laser desorption ionization-time-of-flight mass spectra of the reaction products of phosphatidylethanolamines and hypochlorous acid. Anal. Biochem. 2008, 376, 157-159.
10. Jaskolla, T. W.; Lehmann, W. D.; Karas, M. 4-Chloro- $x$-cyanocinnamic acid is an advanced, rationally designed MALDI matrix. Proc. Natl. Acad. Sci. U.S.A. 2008, 105, 12200-12205.

11. Frisch, M. J.; Pople, J. A.; Binkley, J. S. Self-consistent molecular orbital methods 25. Supplementary functions for Gaussian basis sets. J. Chem. Phys. 1984, 80, 3265-3269.

12. Andersson, M. P.; Uvdal, P. New scale factors for harmonic vibrational frequencies using the B3LYP density functional method with the Triple- $\xi$ basis set $6-311+G(d, p)$. J. Phys. Chem. A 2005, 109, 2937-2974.

13. Richter, G.; Schober, C.; Sü $\beta$, R.; Fuchs, B.; Müller, M.; Schiller, J. The reaction between phosphatidylethanolamines and $\mathrm{HOCl}$ investigated by TLC: Fading of the dye primuline is induced by dichloramines. J. Chromatogr. B 2008, 867, 233-237.

14. Morris, J. C. The acid ionization constant of $\mathrm{HOCl}$ from 5 to $35^{\circ}$. J. Phys. Chem. 1966, 195, 133-140.

15. Glückmann, M.; Karas, M. The initial velocity and its dependence on matrix, analyte, and preparation method in ultraviolet matrix-assisted laser desorption/ionization. J. Mass Spectrom. 1999, 34, 467-477.

16. Juhasz, P.; Vestal, M. L.; Martin, S. A. On the initial velocity of ions generated by matrix-assisted laser desorption ionization and its effect on the calibration of delayed extraction time-of-flight mass spectra. J. Am. Soc. Mass Spectrom. 1997, 8, 209-217.

17. Jaskolla, T. W.; Karas, M. Using fluorescence dyes as a tool for analyzing the MALDI process. J. Am. Soc. Mass Spectrom. 2008, 19, 918-925.

18. Harvey, D. J. Matrix-assisted laser desorption/ionization mass spectrometry of phospholipids. J. Mass Spectrom. 1995, 30, 1333-1346.

19. Fuchs, B.; Schober, C.; Richter, G. Süß, R.; Schiller, J. MALDI-TUF MS of phosphatidylethanolamines: different adducts cause different post source decay (PSD) fragment ion spectra. J. Biochem. Biophys. Methods. 2007, 70, 689-692.

20. Gidden, J.; Liyanage, R.; Durham, B.; Lay J. O. Jr., Reducing fragmentation observed in the matrix-assisted laser desorption/ionization timeof-flight mass spectrometric analysis of triacylglycerols in vegetable oils. Rapid Commun. Mass Spectrom. 2007, 21, 1951-1957.

21. Thomas, E. L.; Grisham, M. B.; Jefferson, M. M. Preparation and characterization of chloramines. Methods Enzymol. 1986, 132, 569-585.

22. Ohanessian, G. Interaction of MALDI matrix molecules with $\mathrm{Na}^{+}$in the gas phase. Int. J. Mass Spectrom. 2002, 219, 577-592.

23. Chinthaka, S. D. M.; Rodgers, M. T. Potassium ion affinities of commonly used MALDI matrices: Determined by guided ion beam tandem mass spectrometry. Proceedings of the 55th ASMS Conference on Mass Spectrometry and Allied Topics; Indianapolis, IN, June, 2007.

24. Schulz, E.; Karas, M.; Rosu, F.; Gabelica, V. Influence of the matrix on analyte fragmentation in atmospheric pressure MALDI. J. Am. Soc. Mass Spectrom. 2006, 17, 1005-1013.

25. Gabelica, V.; Schulz, E.; Karas, M. Internal energy build-up in matrixassisted laser desorption/ionization. J. Mass Spectrom. 2004, 39, 579-593.

26. Karas, M.; Bahr, U.; Fournier, I.; Glückmann, M.; Pfenninger, A. The initial-ion velocity as a marker for different desorption-ionization mechanisms in MALDI. Int. J. Mass Spectrom. 2003, 226, 239-248. 